\title{
Energy dependence of optical potential in the near barrier elastic scattering of ${ }^{11} \mathbf{B}$ from ${ }^{232} \mathrm{Th}$
}

\author{
Shradha Dubey 1,2 , S. Mukherjee ${ }^{1}$, D. Patel ${ }^{1}$, Y. K. Gupta ${ }^{2}$, L. S. Danu ${ }^{2}$, B. N. Joshi ${ }^{2}$, G. K. Prajapati ${ }^{2}$, S. \\ Mukhopadhyay², B. V. J ohn², B. K. Nayak², and D. C. Biswas ${ }^{2, a}$ \\ ${ }^{1}$ Physics Department, Faculty of Science, M.S. University of Baroda, Vadodara-390002, India \\ ${ }^{2}$ Nuclear Physics Division, Bhabha Atomic Research Centre, Mumbai - 400085
}

\begin{abstract}
The elastic scattering cross sections of ${ }^{11} \mathrm{~B}$ projectile on the ${ }^{232} \mathrm{Th}$ target have been measured at different bombarding energies close to the Coulomb barrier. The data has been analyzed for this system using the optical model ECIS code with phenomenological Woods-Saxon forms of the optical potentials. The energy dependence of the volume type real and imaginary parts of the optical potentials are derived from the best fit of the experimental angular distribution data. The total reaction cross sections are obtained from optical model analysis.
\end{abstract}

\section{Introduction}

The rich interplay between the intrinsic structure and the reaction dynamics of the interacting nuclei has been a hall-mark of near-barrier heavy-ion interactions [1]. One of the manifestations of this feature is a strong energy dependence of the optical model potential deduced from analyses of elastic scattering angular distributions at energies around the Coulomb barrier [1, 2]. The study of elastic scattering angular distributions determine parameters of the real and imaginary parts of the nuclear interaction potential. From systematic analysis of elastic scattering measurements involving tightly bound nuclei, the so called "threshold anomaly" (TA) has been observed in a number of systems [1]. This has been understood in terms of couplings of elastic channel to the direct reaction channels that generate an additional attractive real dynamic polarization potential. The study of the TA is important to investigate the influence of the breakup and other reaction mechanisms on the elastic and fusion channels [3-5].

In the present work, the elastic scattering angular distribution measurements have been carried out for ${ }^{11} \mathrm{~B}+$ ${ }^{232} \mathrm{Th}$ system at energies from $4 \%$ below the Coulomb barrier $\left(\mathrm{V}_{\text {lab }} \sim 54 \mathrm{MeV}\right)$ to approximately $20 \%$ above the barrier. The total reaction cross sections for this system have also been derived to understand the role of projectile breakup on the total reaction cross sections. The present article has been organized in the following way. The experimental set up is described in Sec.2. Data analysis using the Wood-Saxon potential (WSP) to determine the energy dependence of potential parameters have been dis-

${ }^{\mathrm{a}}$ corresponding author:dcbiswas@barc.gov.in cussed in Sec.3. In Sec.4, a systematic study of total reaction cross section for ${ }^{11} \mathrm{~B}+{ }^{232} \mathrm{Th}$ system has been discussed. In Sec.5, the summary and conclusions of the present work are reported.

\section{Experimental procedure}

The experiment was performed at the 14UD BARC-TIFR Pelletron facility at Mumbai using beams of ${ }^{11} \mathrm{~B}$ in a wide energy range around the Coulomb barrier, i.e., 52, 53, 54, $55,56,57,59,61$ and $65 \mathrm{MeV}$ for ${ }^{11} \mathrm{~B}+{ }^{232} \mathrm{Th}$ system. The observed uncertainty in the beam energy was about $1 \%$ for all the selected energies. A self supporting ${ }^{232} \mathrm{Th}$ target of thickness $1.5 \mathrm{mg} / \mathrm{cm}^{2}$ was placed at the center of the general purpose scattering chamber and the elastically scattered ${ }^{11} \mathrm{~B}$ particles were detected by silicon $\Delta \mathrm{E}$-E telescopes mounted on a movable arm of the chamber. Four telescopes of thickness $\left(\mathrm{T}_{1}\right)$ with $\Delta \mathrm{E}=25 \mu \mathrm{m}$ and $\mathrm{E}=$ $300 \mu \mathrm{m},\left(\mathrm{T}_{2}\right)$ with $\Delta \mathrm{E}=40 \mu \mathrm{m}$ and $\mathrm{E}=300 \mu \mathrm{m},\left(\mathrm{T}_{3}\right)$ with $\Delta \mathrm{E}=25 \mu \mathrm{m}$ and $\mathrm{E}=300 \mu \mathrm{m}$ and $\left(\mathrm{T}_{4}\right)$ with $\Delta \mathrm{E}=25 \mu \mathrm{m}$ and $\mathrm{E}=300 \mu \mathrm{m}$ were used in the experiment. The detector telescopes were placed at an angular separation of $10^{\circ}$ and two silicon detectors with thickness around $300 \mu \mathrm{m}$ were mounted at fixed angles $\pm 18^{\circ}$ with respect to beam direction for absolute normalization and beam monitoring. The angular distributions were measured in steps of $5^{\circ}$ in the angular range from $35^{\circ}$ to $170^{\circ}$. The uncertainty on the angular range of each telescope was $\pm 0.81^{\circ}$.

\section{Optical Model Analysis Of Elastic Scattering}

The optical model fits to the elastic scattering data have been performed using the ECIS code [6]. The phe- 
nomenological Woods-Saxon form of the interaction potential with only volume terms have been used in the analysis. To obtain the starting parameters, a global best fit procedure for all energies were performed, using the three parameters characterized by real and imaginary depth of the potential, reduced radii $\left(\mathrm{r}_{o}\right)$ and diffuseness $\left(\mathrm{a}_{v}\right.$ and $a_{w}$ ). Thereafter, in order to avoid a fit procedure with too many parameters, the real and imaginary reduced radii were fixed at $1.06 \mathrm{fm}$ for the ${ }^{11} \mathrm{~B}+{ }^{232} \mathrm{Th}$ system, similar to the value used earlier $[4,5]$. Using this radius, and varying the diffuseness parameters $\mathrm{a}_{v}$ and $\mathrm{a}_{w}$ (real and imaginary respectively) within the interval from $0.67 \mathrm{fm}$ to $0.75 \mathrm{fm}$, an attempt was made to obtain the best possible parameters of the optical potential that best describe the elastic scattering angular distribution. In the present work, the best possible fitted values were obtained for $\mathrm{a}_{v}, \mathrm{a}_{w}=0.71 \mathrm{fm}$. The real and imaginary radius parameters $\left(\mathrm{r}_{o}=1.06 \mathrm{fm}\right)$ and diffuseness parameters $\left(\mathrm{a}_{v}, \mathrm{a}_{w}=0.71 \mathrm{fm}\right)$ were fixed for all energies. The depths of real and imaginary potentials were varied to obtain minimum value of $\chi^{2}$ for the ${ }^{11} \mathrm{~B}+{ }^{232} \mathrm{Th}$ system.

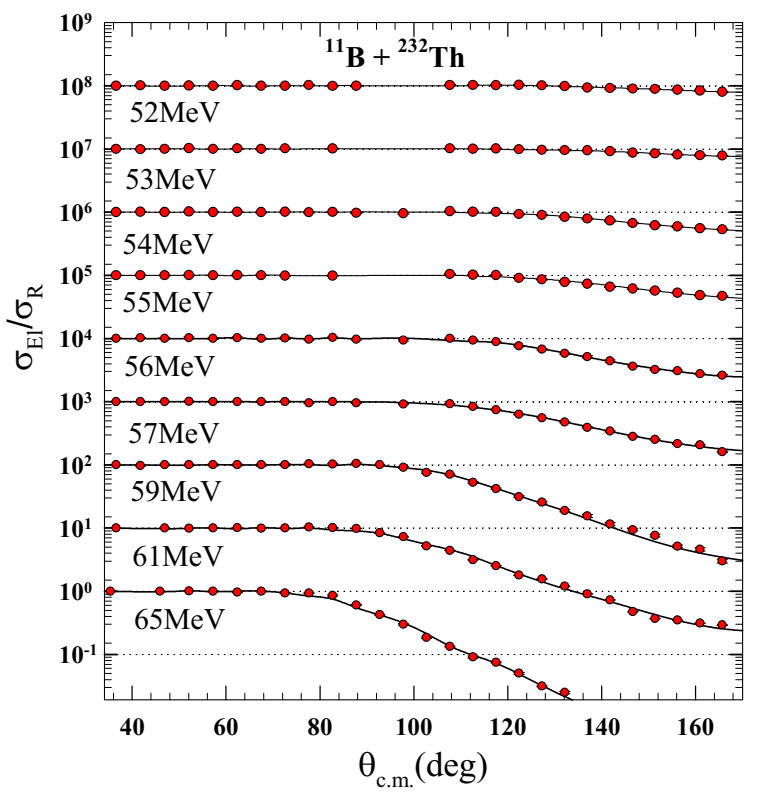

Figure 1. Experimental elastic scattering cross section normalized to the Rutherford cross section as a function of $\theta_{c . m}$. for ${ }^{11} \mathrm{~B}$ $+{ }^{232}$ Th system.

The potential parameter values for the best fit and the total reaction cross sections are shown in Table-I. The best fit optical model parameters show significant energy dependence as reflected from Table-I, which is a characteristic feature of the elastic scattering. The solid lines in Fig. 1 show the best fit of the experimental data for the elastic scattering angular distributions for ${ }^{11} \mathrm{~B}+{ }^{232} \mathrm{Th}$ system.

To reduce the ambiguities of the best fitted parameters of the optical potential, the strong sensitive radius $\mathrm{R}_{S r}$ and $\mathrm{R}_{S i}$ corresponding to the real and imaginary potential were determined. For this purpose the radius parameters were kept fixed and the depth parameters of the real and imagi-
Table 1. Optical model parameters obtained by fitting the experimental elastic differential cross section data using the ECIS code for ${ }^{11} \mathrm{~B}+{ }^{232} \mathrm{Th}$ system.

\begin{tabular}{|l|l|l|l|l|}
\hline $\mathrm{E}_{\text {lab }}(\mathrm{MeV})$ & $\mathrm{V}_{r}(\mathrm{MeV})$ & $\mathrm{V}_{i}(\mathrm{MeV})$ & $\frac{\chi^{2}}{n}$ & $\sigma_{R}(\mathrm{mb})$ \\
\hline \hline 52 & 194.0 & 0.131 & 1.582 & 7.02 \\
53 & 149.2 & 15.03 & 0.640 & 29.81 \\
54 & 162.0 & 31.43 & 1.210 & 89.74 \\
55 & 134.2 & 27.32 & 4.060 & 105.4 \\
56 & 126.0 & 51.44 & 3.523 & 216.2 \\
57 & 109.4 & 57.02 & 4.700 & 279.7 \\
59 & 121.5 & 59.23 & 4.900 & 432.6 \\
61 & 73.67 & 89.30 & 11.21 & 601.7 \\
65 & 53.45 & 109.7 & 9.210 & 886.4 \\
\hline
\end{tabular}

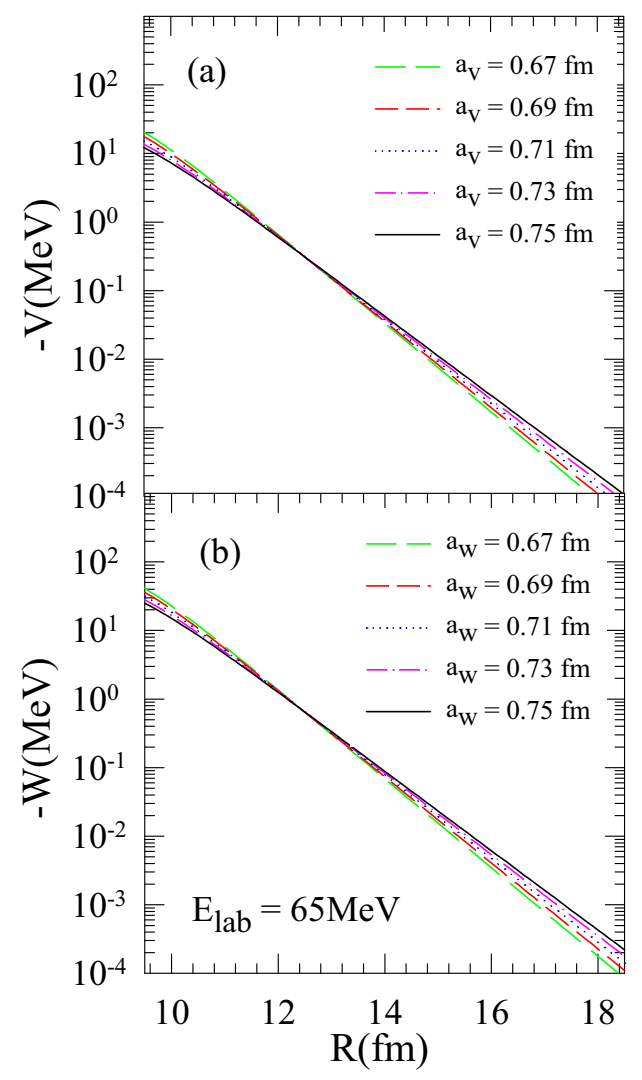

Figure 2. Several potential that produce similar fits of the data, for $65 \mathrm{MeV}$. The crossing point are the derived real (a) and imaginary (b) sensitivity radii for ${ }^{11} \mathrm{~B}+{ }^{232} \mathrm{Th}$ system.

nary potentials were fitted by varying the diffuseness from 0.67 to $0.75 \mathrm{fm}$, in step of $0.02 \mathrm{fm}$ for all energies of ${ }^{11} \mathrm{~B}$ $+{ }^{232} \mathrm{Th}$ system. The strong sensitive radii [8] were determined, where the real and imaginary part of different optical potentials that fitted the data cross each other as shown in Fig. 2 (a) and 2(b) for $65 \mathrm{MeV}$ for ${ }^{11} \mathrm{~B}+{ }^{232} \mathrm{Th}$ system. The real sensitive radii values were observed to be in the range of 12.4 to $11.3 \mathrm{fm}$ with a average of $\mathrm{R}_{S r}=$ $11.57 \mathrm{fm}$ and for the imaginary sensitive radii values range from 12.7 to $13.5 \mathrm{fm}$ with a average value of $\mathrm{R}_{S i}=13.34$ $\mathrm{fm}$. A mean sensitive radius of $\mathrm{R}_{s}=12.45 \mathrm{fm}$ for ${ }^{11} \mathrm{~B}+$ ${ }^{232} \mathrm{Th}$ system (average between $\mathrm{R}_{S r}=11.57 \mathrm{fm}$ and $\mathrm{R}_{S i}$ 
$=13.34 \mathrm{fm}$ ) was obtained to derive energy dependence of real and imaginary potential. In the analysis for this system the values of radius parameters for real and imaginary parts were kept fixed at $1.06 \mathrm{fm}$ for all the calculations. A similar fitting procedure can also be found in the literature $[2,7]$ for ${ }^{11} \mathrm{~B}+{ }^{209} \mathrm{Bi}$ system. The corresponding values of the energy dependence of the real and imaginary potentials for the ${ }^{11} \mathrm{~B}+{ }^{232} \mathrm{Th}$ system are shown in Fig. 3 . The error bars in Fig. 3 represent the range of deviation of the potential corresponding to a $\chi^{2}$ variation of one unit.

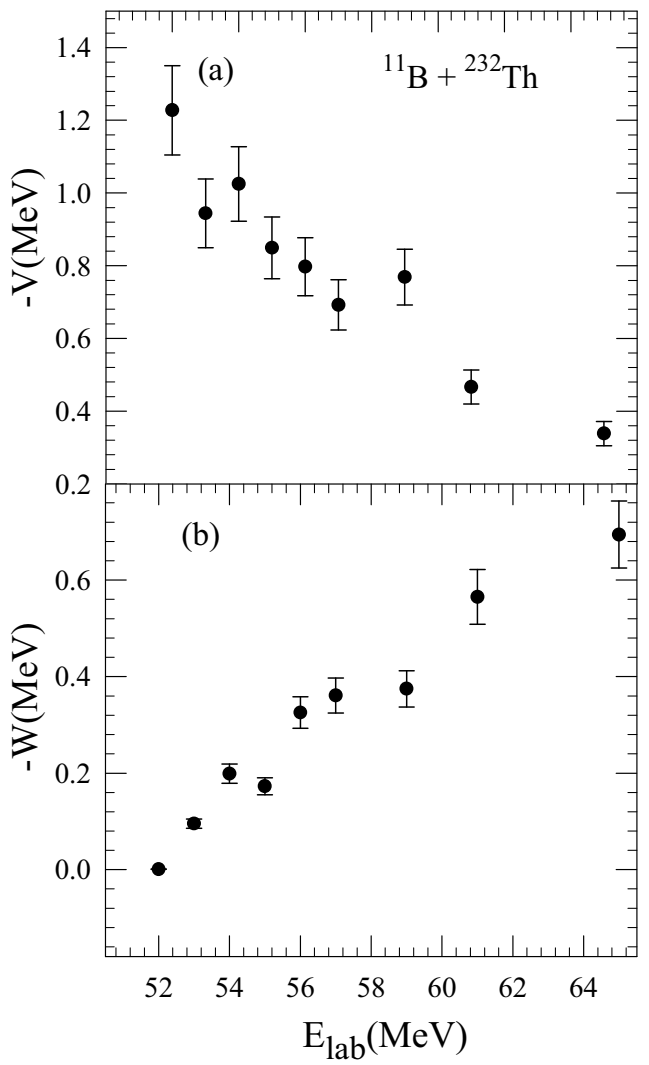

Figure 3. Energy dependence of the real and imaginary parts of the optical potential obtained for the ${ }^{11} \mathrm{~B}+{ }^{232} \mathrm{Th}$ system at an average radius $R_{s}=12.45 \mathrm{fm}$.

\section{Total Reaction Cross Section}

The fusion cross sections have been calculated for ${ }^{11} \mathrm{~B}+$ ${ }^{232}$ Th system by using CCFULL code [9]. The energy range used in the calculation was 52 to $65 \mathrm{MeV}$, in steps of $1 \mathrm{MeV}$. In the present work, the total reaction cross sections derived from experimental elastic scattering data analysis for ${ }^{11} \mathrm{~B}+{ }^{232} \mathrm{Th}$ system was compared with the calculated fusion cross sections as shown in Fig. 4 (a). The total reaction cross sections obtained from optical model ECIS code. In order to eliminate the projectile size effect on the reaction cross sections for ${ }^{11} \mathrm{~B}+{ }^{232} \mathrm{Th}$ system, the "reduction" method was used. This method was proposed by Gomes et al. [10] and has been well implemented by others $[4,5,11,12]$. The reduced cross section values were calculated at all energies for the system. In this
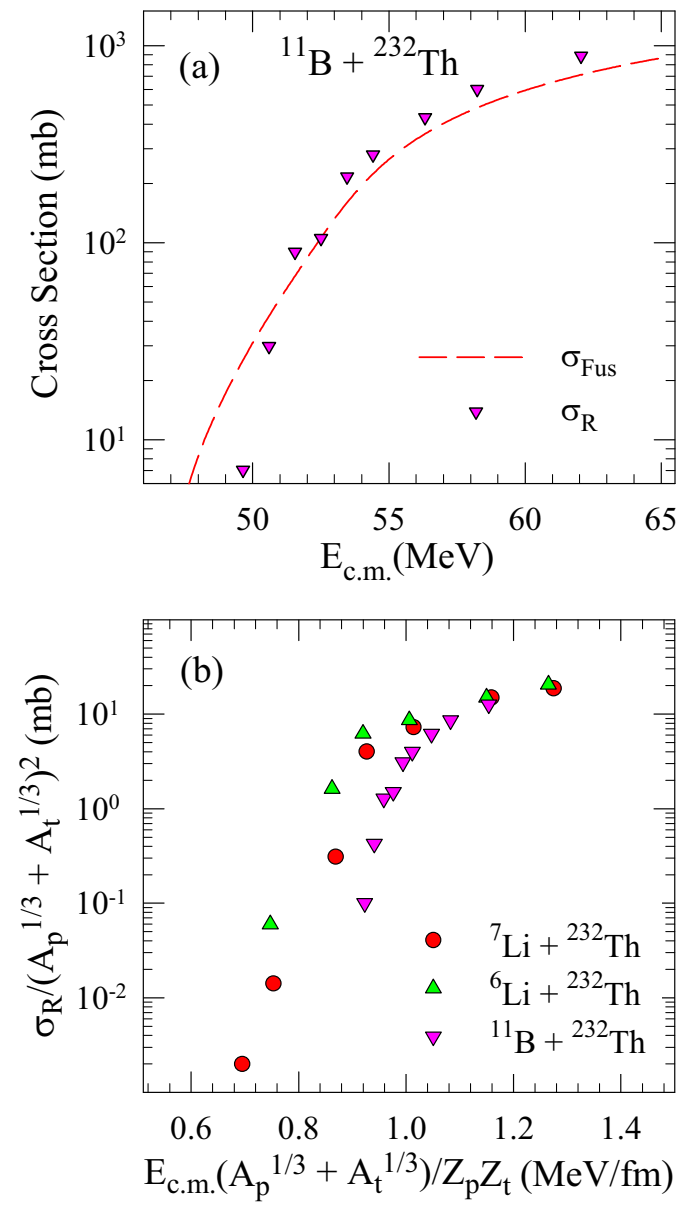

Figure 4. Total fusion cross section ( $\sigma_{\text {fus }}$ ) (by CCFULL) and total reaction cross section $\left(\sigma_{R}\right)$ for ${ }^{11} \mathrm{~B}+{ }^{232} \mathrm{Th}$ system as a function of the bombarding energy in 4 (a). Reduced total reaction cross section vs reduced projectile energy for the ${ }^{6,7} \mathrm{Li}+{ }^{232} \mathrm{Th}$ and ${ }^{11} \mathrm{~B}+{ }^{232} \mathrm{Th}$ reactions using the prescription given in Ref. [10] in 4 (b).

method, the quantities $\sigma_{R} /\left(A_{P}^{1 / 3}+A_{T}^{1 / 3}\right)^{2}$ vs $E_{c . m .}\left(A_{P}^{1 / 3}+\right.$ $\left.A_{T}^{1 / 3}\right) / Z_{P} Z_{T}$ are plotted, where $\mathrm{P}$ and $\mathrm{T}$ represent the projectile and target respectively, and $\sigma_{R}$ is the total reaction cross section. As shown in Fig. 4 (b), it can be seen that total reduced reaction cross section of ${ }^{11} \mathrm{~B}+{ }^{232} \mathrm{Th}$ system is smaller than ${ }^{6,7} \mathrm{Li}+{ }^{232} \mathrm{Th}$ system.

\section{Summary and Conclusions}

The elastic-scattering angular distribution measurements have been carried out for the ${ }^{11} \mathrm{~B}+{ }^{232} \mathrm{Th}$ system at several bombarding energies from below to well above the Coulomb barrier. The experimental data have been analyzed using the ECIS code forms of phenomenological optical potentials. The relevant parameters that gives best fit to the elastic scattering angular distribution, were obtained through a $\chi^{2}$-minimization procedure. The enhanced reaction cross sections have been observed at sub-barrier energies for ${ }^{6,7} \mathrm{Li}+{ }^{232} \mathrm{Th}$ systems [5] in comparison to the ${ }^{11} \mathrm{~B}$ $+{ }^{232} \mathrm{Th}$ system. This may be an indication that ${ }^{11} \mathrm{~B}$ has a lower breakup probability as compared to ${ }^{6,7} \mathrm{Li}$ projectiles. 


\section{Acknowledgement}

We are thankful to the operating staff of the BARC-TIFR pelletron, Mumbai, India, for smooth running of the machine during experiment. The authors S.D. and S.M. thank the UGC-DAE-CSR, Kolkata for financial support through a major research project.

\section{References}

[1] G. R. Satchler, Phys. Rep. 199, 147 (1991).

[2] A. Shrivastava, S. Kailas, P. Singh, A. Chatterjee, A. Navin, A. M. Samant, V. Ramdev Raj, S. Mandal, S. K. Datta, S. K. Datta, Nucl. Phys. A 635, 411 (1998).

[3] J. Lubian et al., Nucl. Phys. A 791, 24 (2007).

[4] N. N. Deshmukh et al., Phys. Rev. C 83, 024607 (2011).
[5] Shradha Dubey et al., Phys. Rev. C 89, 014610 (2014).

[6] J. Raynal, Phys. Rev. C 23, 2571 (1981).

[7] S. Y. Lee and W. Y. So, Eur. Phys. J. A 791, 24 (2007).

[8] G. R. Satchler, in Proceeding of the International Confrence on reactions between complex Nuclei, Nashville,TN, edited by R. L.Robinson, F. K. McGowan, J. B. Ball and J. H. Hamilton(North Holland, Amsterdam, 1974, vol. 2, P. 171.

[9] K. Hagino et al., comput. Phys. commun. 123, 143 (1999).

[10] P. R. Gomes, J. Lubian, I. Padron, R. M. Anjos, Phys. Rev. C 71, 017601 (2005).

[11] N. N. Deshmukh et al., Eur. Phys. J. A 47, 118 (2011).

[12] S. Mukherjee et al., Eur. Phys. J. A 45, 23 (2010). 